\title{
Ambulatory diffuse optical tomography and multimodality physiological monitoring system for muscle and exercise applications
}

Gang $\mathrm{Hu}$

Quan Zhang

Vladimir Ivkovic

Gary E. Strangman 


\title{
Ambulatory diffuse optical tomography and multimodality physiological monitoring system for muscle and exercise applications
}

\author{
Gang Hu, ${ }^{a}$ Quan Zhang, ${ }^{\text {a,b }}$ Vladimir Ivkovic, ${ }^{a}$ and Gary E. Strangman ${ }^{a, b, *}$ \\ a Harvard Medical School, Massachusetts General Hospital, Neural Systems Group, Building 149, 13th Street, Charlestown, \\ Massachusetts 02129, United States \\ ${ }^{\mathrm{b} B a y l o r}$ College of Medicine, Center for Space Medicine, 6500 Main Street, Houston, Texas 77030, United States
}

\begin{abstract}
Ambulatory diffuse optical tomography (aDOT) is based on near-infrared spectroscopy (NIRS) and enables three-dimensional imaging of regional hemodynamics and oxygen consumption during a person's normal activities. Although NIRS has been previously used for muscle assessment, it has been notably limited in terms of the number of channels measured, the extent to which subjects can be ambulatory, and/or the ability to simultaneously acquire synchronized auxiliary data such as electromyography (EMG) or electrocardiography (ECG). We describe the development of a prototype aDOT system, called NINscan-M, capable of ambulatory tomographic imaging as well as simultaneous auxiliary multimodal physiological monitoring. Powered by four $A A$ size batteries and weighing $577 \mathrm{~g}$, the NINscan-M prototype can synchronously record 64-channel NIRS imaging data, eight channels of EMG, ECG, or other analog signals, plus force, acceleration, rotation, and temperature for $24+\mathrm{h}$ at up to $250 \mathrm{~Hz}$. We describe the system's design, characterization, and performance characteristics. We also describe examples of isometric, cycle ergometer, and free-running ambulatory exercise to demonstrate tomographic imaging at $25 \mathrm{~Hz}$. NINscan-M represents a multiuse tool for muscle physiology studies as well as clinical muscle assessment. @ 2016 Society of Photo-Optical Instrumentation Engineers (SPIE) [DOI: 10.1117/ 1.JBO.21.9.091314]
\end{abstract}

Keywords: wearable; laser; tissue oxygenation; muscle; diffuse optical imaging.

Paper 160025SSRR received Jan. 19, 2016; accepted for publication Jul. 8, 2016; published online Jul. 28, 2016.

\section{Introduction}

Ambulatory near-infrared spectroscopy (aNIRS) and ambulatory diffuse optical tomography (aDOT) enable a user to wear a near-infrared spectroscopy (NIRS) device for noninvasive, continuous monitoring, or imaging of tissue oxygenation and hemodynamics with minimal restrictions on their activities. Wearable devices are rapidly expanding from the traditional electrocardiography (ECG) monitoring ${ }^{1}$ and electroencephalography $(\mathrm{EEG})^{2,3}$ to pulse oximetry, blood pressure, and various metrics of activity and exercise. ${ }^{4-7}$ NIRS is distinct from these other modalities in that it can monitor tissue blood oxygenation and oxygen utilization. These are particularly useful capabilities for muscle assessment during and following exercise to evaluate muscle oxygenation and reserve, ${ }^{8,9}$ muscle training, ${ }^{10,11}$ and to evaluate certain disease states or recovery processes. ${ }^{12-15}$

A number of portable NIRS instruments have previously been developed, enabling studies on progressively more mobile subjects. ${ }^{16,17}$ Runman and Micro Runman were pioneering developments in the laboratory of Chance et al., ${ }^{18}$ which first demonstrated NIRS portability. These were followed by wearable, battery powered systems, ${ }^{19}$ hematoma detection systems, ${ }^{20}$ and more recently the wireless system of Wolf et al., ${ }^{17,21}$ the PortaMon system, ${ }^{22}$ plus our own NINscan2a system. ${ }^{23}$ These devices all support only a small number of channels $\quad<10$ measurements each). Atsumori et al. $^{24,25}$ further described a

*Address all correspondence to: Gary E. Strangman, E-mail: strang@ nmr.mgh. harvard.edu wearable optical topography system with eight source locations and eight detectors that can generate two-dimensional (2-D) images of the entire forehead at frame rates up to $5 \mathrm{~Hz}$.

Despite such advancements in portable NIRS technology, significant gaps remain, particularly for muscle applications. Although wireless monitoring has considerable appeal, it also has drawbacks in terms of communication reliability (packet dropping), security/privacy, and restrictions on the distance the subject can travel from the receiving base station (typically about $10 \mathrm{~m}$ ). Also important for muscle applications, exercise does not simply generate physiological changes localized to the monitored muscle. Exercise also leads to multiple systemic physiological changes, including heart rate, respiration rate, skin perfusion, blood pressure, and various other alterations known to affect NIRS signals. Unfortunately, most aNIRS devices are not designed to collect such systemic physiological measurements, which can be important for appropriate interpretation of aNIRS recordings.

Exercise also affects multiple muscle groups - at varying tissue depths-and aNIRS systems are rarely designed for imaging in depth or imaging at all. ${ }^{26}$ Imaging is important because oxygen use has been shown to vary significantly in different parts of the same muscle in various exercise settings. ${ }^{27-29}$ Such heterogeneous oxygen utilization is not restricted to exercise but has been observed during passive stretch ${ }^{30}$ and even at rest. ${ }^{31}$ Thus, the hemodynamic status of a muscle is not well represented by a single-point measurement within that muscle. This highlights

$1083-3668 / 2016 / \$ 25.00$ (c) 2016 SPIE 
the importance of being able to obtain spatially resolved measurements, both in-plane and in-depth. Understanding the spatial heterogeneity of muscle hemodynamics not only informs basic muscle physiology and potentially help improve muscle training paradigms, ${ }^{32}$ but also could support clinical decision making. For example, a recent study demonstrated significant and clinically important spatial variations in foot perfusion using optical tomography that could help guide treatment toward the affected vascular beds. ${ }^{33}$ Although ambulatory NIRS is not required for that particular clinical application, an ambulatory system can facilitate research or clinical assessments in a wider variety of settings than traditional systems, including during exercise.

We have focused in particular on developing wearable tissue imaging capabilities designed for astronauts during spaceflight. Such devices are required to be ultraportable, easy to use, as well as multiuse, i.e., any given device requires multiple capabilities suitable for various applications in remote and low-resource settings. For NIRS, this means any deployed device must be suitable for imaging more than just one body part, compatible with more than one probe geometry, and provide more than NIRSonly measurements. Such design requirements inherently lead toward a highly portable, general-purpose device suitable for many Earth-based applications as well.

A particularly important application in spaceflight is muscle assessment. Without countermeasures, microgravity quickly leads to reduced muscle (and bone) mass, ${ }^{34}$ reduced strength, ${ }^{35}$ and reduced endurance. ${ }^{36}$ To address this problem, each astronaut is typically scheduled to perform exercise countermeasures for 1.5 to $2 \mathrm{~h}$ every day, including treadmill running, cycle ergometry, and heavy-resistance exercise. Although the current countermeasure approach has proven helpful, ${ }^{37}$ it is also a major time commitment. Consequently, methods for more accurately tracking muscle status and optimizing training regimens are of interest. Given the wealth of prior NIRS work in exercise science, e.g., ${ }^{38-40}$ a flexible and multimodal, ambulatory tomography device has strong potential to fill this gap.

We previously developed and demonstrated 4-channel aNIRS technologies in extreme/low-resource environments including parabolic flight and high-altitude hiking. ${ }^{23}$ Here, we present the development of a 64-channel ambulatory tomography (aDOT) prototype designed for $25-$ to $250-\mathrm{Hz}$ frame rate use and as a fully wearable, lightweight, and multipurpose device supporting up to 14 auxiliary sensors/inputs. This multimodality capability in fact achieves many of the requirements of the "ideal imager" for NIRS-based muscle assessment as described by Ferrari et al. ${ }^{16}$ This paper focuses on the hardware development plus example applications related to muscle and physiological monitoring.

\section{Multimodal Ambulatory Instrumentation Design}

\subsection{General Description and Features}

NINscan-M ( $\mathrm{M}=$ multiuse) is a continuous-wave NIRS prototype designed for the spaceflight demands of high mobility, automation, and flexibility. It consists of a recording box plus sensors, which can be powered by wall power or support continuous recordings for up to $26 \mathrm{~h}$ on four rechargeable AA batteries, sampling at up to $250 \mathrm{~Hz}$. NINscan-M includes eight laser diode sources and eight photodiode detectors for aDOT, deployable in arbitrary geometries for limb, head/brain, or even abdominal applications. The NIRS data collection process utilizes a time-division multiplexing (TDM) scheme combined with autobaselining. In this scheme, one source is turned on at a time during which "active" measurements are collected from all eight detectors. Then all sources are turned off and "baseline" measurements are collected from all detectors. This process is then repeated for each of the remaining sources. The final NIRS measurement is computed as the difference between active minus baseline measures, thereby nulling ambient lighting conditions as well as accounting for dark current.

In addition to the 64 NIRS channels, NINscan-M also supports up to eight gain-controllable 16-bit analog inputs compatible with electromyography (EMG), ECG, electrooculography (EOG), EEG, or other analog signal sources, one 12-bit analog input, and plus four digital sensor inputs. Two additional digitalin/-out lines are available for synchronizing with other devices, and four user-input buttons provide a method for marking external events directly in the data stream. Bluetooth wireless broadcast of acquired data can be turned on/off, and other device configuration parameters can be set via a human-readable text file. Device deployment was designed to be as simple as possible: position the sensors and flip the single on/off power switch. When recording, the CPU continuously stores data on an secure digital (SD) flash memory card, where a $32-\mathrm{Gb}$ card can store data for $\sim 215 \mathrm{~h}$ of continuous recording. The device can be turned off at any time (which automatically closes the data file) and restarted as needed without first offloading existing data (data are saved to a newly timestamped file). Data transfer is achieved via the SD card. The prototype is enclosed in a custom acrylonitrile butadiene styrene three-dimensional (3-D)-printed box with a volume of $555 \mathrm{~cm}^{3}$ and mass of $577 \mathrm{~g}$ (including batteries). The functional structure of the prototype appears in Fig. 1 and a photo appears in Fig. 2(a).

\subsection{Near-Infrared Spectroscopy Subcomponent}

For light sources, NINscan-M currently uses dual-wavelength laser diodes (Axcel Photonics Inc., Marlborough, Massachusetts). Detectors are monolithic silicon photodiodes with a on-chip transimpedance amplifier (OPT101; Texas Instruments Inc, Dallas, Texas). Despite the substantial number of sources and detectors, the NIRS sensor pads are designed for low-profile and motionrobust use, as in our previous designs. ${ }^{23}$ In particular, we used a lightweight Velcro-based form to hold the laser diodes and photodiodes. A medical tape layer is used for easier cleaning, and light shaping diffusers are attached to the surface of the laser diodes to reduce the effective laser class to class 1 (eye-safe). In certain applications, we affix small $(3 \times 5 \mathrm{~mm})$ coated lenses (Edmunds Optics, Inc.) to the optical components to improve coupling, particularly for applications through thick hair on the head. The portions of the sensor that contact the subject are either glass, polycarbonate, medical tape, or nylon, for broad biocompatibility.

Gain control within the NIRS system is largely automated and includes three separate stages. First, laser source power is controlled digitally, currently via configuration file settings. This provides 256-step control of output power for each source from 0 - to $10-\mathrm{mW}$ optical power, thereby enabling system deployment with either small or large targets (e.g., animals versus adult humans) with fewer concerns about signal ceiling or floor effects on recorded signals. Second, we embedded digitally controlled detector gain control within the sensor itself. This in-probe gain stage currently provides two sensitivity levels (low and high, or $1 \times$ and $75 \times$ ) to accommodate detectors that are positioned close to a laser source (e.g., $\sim 1 \mathrm{~cm}$, for measuring 


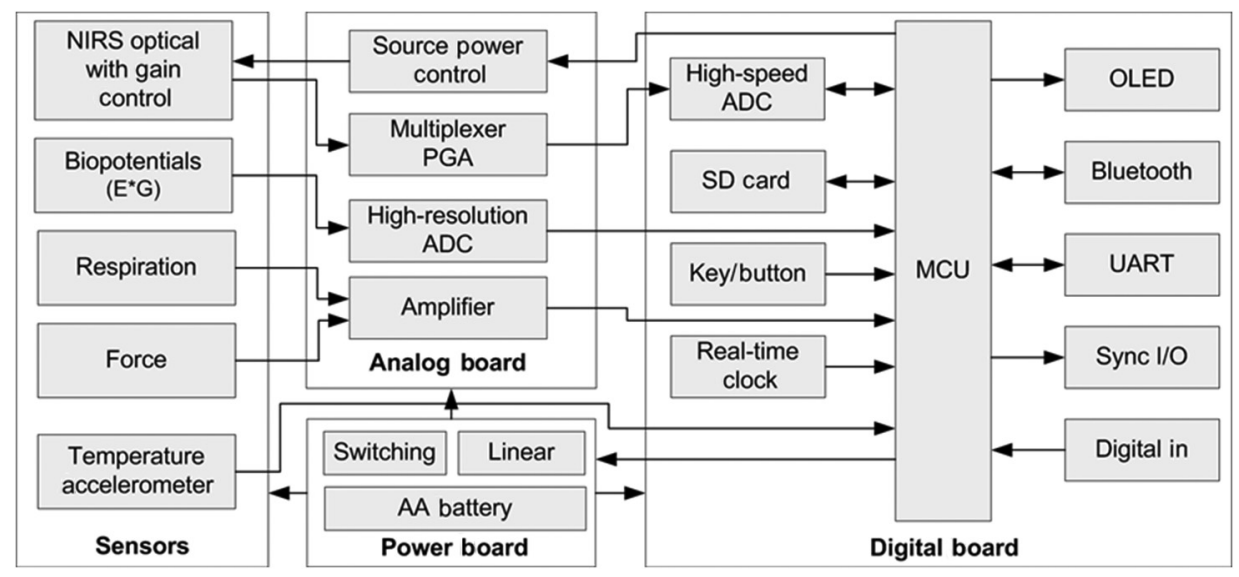

Fig. 1 Functional diagram of NINscan-M. The NIRS block contains all aDOT sensor components. The analog board supports all gain controls for NIRS plus biopotential A/D sampling. The system is controlled by the microcontroller unit on the digital board.

fat or skin layer interference) $)^{41-46}$ or farther from a source (for deeper measurements), respectively. These in-sensor gains can be determined automatically when the instrument is first turned on and then held fixed for the remainder of that recording or changed dynamically depending on which source is currently illuminated (configurable).

The third gain stage is located inside the control box and is always dynamic in nature. A total of 64 gains are encoded, one for each source-detector pair, with 20 unique gain settings for each (up to a maximum of $1024 \times$ amplification). When the device is first switched on, the system illuminates the first source and determines optimal gain settings for each detector paired with that source. The device then switches on the second source, determining a new set of eight optimal detector gain settings, and so on for the remaining sources. These 64 gain values are then stored and dynamically deployed during recording, depending on which source is currently active. This design enables the system to optimize signal levels for each source-detector pair in tomographic imaging geometries that include multiple source-detector distances (and hence dramatically different light input levels) seen by a given detector. Overall, this unique three-tier system provides a potential dynamic range increase of over 300,000-fold.

\subsection{Near-Infrared Spectroscopy/Diffuse Optical Tomography Sensors}

Given the gain design aforementioned, the system can be utilized with nearly arbitrary probe configurations. For example, our previous NINscan devices included detectors positioned at 15 - and 40-mm distant from the dual-color source. ${ }^{23}$ A single NINscan-M sensor was developed to simultaneously support four such pads [see Fig. 2(b)]. This geometry enables simultaneous monitoring of multiple regions of the body (e.g., multiple muscles), and also affords multidistance measurements to help correct NIRS measurements for superficial layer interference. ${ }^{41-}$ ${ }^{46}$ Given the long-distance detector position $(40 \mathrm{~mm})$ in this sensor, we configure the device to use a higher source power. To avoid saturation of the close detector, a low on-sensor gain setting is automatically selected by the system. The automatically selected control box gain fine-tunes the overall gain to further optimize use of the 16-bit dynamic range of the NIRS analog-todigital (A/D) converter.

Alternatively, the optical components can be reconfigured into arbitrary tomographic imaging geometries. Figure 2(c) shows an example diffuse optical tomography (DOT) sensor using a "strip imaging" geometry. This geometry includes a
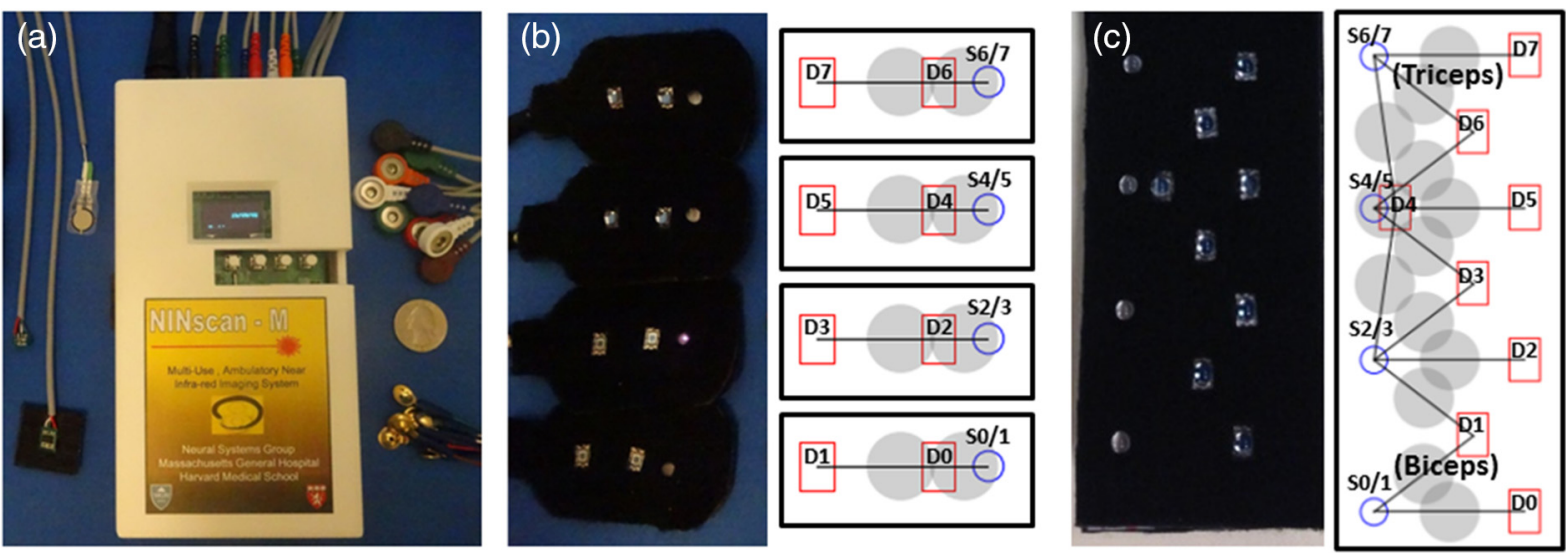

Fig. 2 (a) Photo of NINscan-M prototype and a suite of compatible non-NIRS sensors, (b) photo and illustration of multipoint (and multidistance) "quad" NIRS sensor, (c) photo and illustration of an ambulatory DOT "strip imaging" sensor for NINscan-M. (Biceps and triceps labels refer to the isometric muscle test.) 
line or sources and two lines of detectors: one $25 \mathrm{~mm}$ and another $33 \mathrm{~mm}$ from the sources. We have additionally added a nearby detector (D4) to provide the ability to measure signals in overlying tissue layers (skin and fat). Source-detector separations in this imaging sensor range from $12 \mathrm{~mm}$ (for measuring systemic hemodynamics ${ }^{41,42}$ ), to over $55 \mathrm{~mm}$ for third-nearest neighbors. Using this sensor or the quad sensor only requires plugging it in. The system automatically adjusts gain settings according to the signal-to-noise characteristics for each source-detector pair and dynamically deploys those during recording.

\subsection{Auxiliary Sensors}

A key aspect of NINscan-M is to support a wide range of auxiliary sensors. Such sensors, coupled via an on-device plugpanel, can help provide a more comprehensive assessment of an individual's physiological state and responses to tasks, stimuli, or the environment. The auxiliary sensors can be divided into three categories: (1) high-resolution EMG/ECG/ EOG/EEG analog inputs (collectively referred to as biopotential inputs), (2) digital sensors, and (3) standard analog sensors. Up to eight differential biopotential sensors are supported via a lownoise high-performance integrated A/D chip (ADS1299, Texas Instruments), which provides 24-bit resolution, 250-Hz sample rates and gain settings for each channel. The ADS1299 has a built-in programmable-gain amplifier (PGA), internal reference, and an onboard oscillator. It also comes with a flexible input multiplexer that can be independently connected to the internally generated signals for test, temperature, and lead-off detection. ADS1299 incorporates all commonly required features for portable electrophysiological data collection applications. In NINscan-M, these biopotential inputs can be configured for different types of signals depending on the application, including EMG for sports physiology applications, ECG for chronic heart failure patients, and so on.

NINscan-M's digital auxiliary sensors (based on the $\mathrm{I}^{2} \mathrm{C}$ digital bus and protocol) include accelerometers, gyroscope sensors, and temperature sensors. Accelerometers (ADXL345 and ADXL375, Analog Devices, Inc) and gyroscope sensors (L3GD20H, STMicroelectronics, Inc.) provide important postural information and quantitative measurements of motion and activity levels. Movements indicated by accelerometers can also help with identification and removal of motion artifacts and help substantiate a log of activities. Since ambient and skin temperature play important roles for skin perfusion ${ }^{47,48}$-a major interference factor that affects noninvasive NIRS measurements-we also added temperature sensor (AD7415, Analog Devices, Inc.). NINscan-M can support up to a practical maximum of $\sim 6$ digital sensors on the $\mathrm{I}^{2} \mathrm{C}$ bus, depending on the sampling-rate needs of each device.

Finally, the microcontroller itself has a built-in A/D capability, supporting up to eight data inputs, a 12-bit resolution, and a 250-Hz sampling rate. To date, we have integrated a force sensor (with gain and bias control) and an analog respiration-induced plethysmography sensor through these analog lines. Altogether, NINscan-M has the potential to sample 64 NIRS signals, plus 8 biopotential, $\sim 6$ digital inputs, and 1 to 8 lower-resolution analog channels, all synchronized at $250 \mathrm{~Hz}$. In addition, two separate digital-input and -output lines (0 to $3.3 \mathrm{~V}$ ) are provided for interdevice synchronization.

\subsection{Controller and Configuration}

NINscan-M is driven by an STM32 F4 microcontroller and clocked by the interrupt signal generated from biopotential chip ADS1299. The microcontroller performs the initial gainsetting procedure, conducts all data acquisition, and drives the organic light-emitting diode display along with the digitalinput/-output synchronization. The device also supports four user buttons (expandable to eight), which enable users to insert up to eight distinct event-markers during operation, with the same 4-ms resolution as all other data channels. For configuration, when the power switch is turned on, the system first loads a default configuration file. This human-editable text file configures system operation including source powers, biopotential gain settings, force-channel amplification setting, enabling/disabling of the digital input/output capabilities, the on-sensor gain switching mode, and Bluetooth transmit. If Bluetooth is enabled, the microcontroller broadcasts the NIRS data in real time for viewing via a graphical interface on a separate computer.

\subsection{Characterization Testing}

Electronic and phantom tests were performed to characterize the performance of the NINscan-M system similar to previous work. ${ }^{23}$ Optical probes were secured on four homogeneous phantoms with wide-ranging optical properties (from light to dark) to provide the signal span and help estimate parameters such as noise equivalent power (NEP) and dynamic range. A 24-h continuous data collection on the phantoms was used to estimate the system drift.

\subsection{Isometric, Dynamic, and Ambulatory Muscle Applications}

To demonstrate the capabilities of NINscan-M and its suitability for muscle applications, we conducted the tests described as follows. All procedures were approved by the Massachusetts General Hospital, Institutional Review Board (IRB) that oversees human subject testing.

Isometric muscle oxygenation time series imaging: One healthy subject (female, age 22) was recruited for this test. EMG sensors were positioned on the upper right arm, over distal and proximal aspects of biceps brachii, triceps brachii, and one over the body of flexor carpal radii (fCR). A single ECG lead was attached at position V4, with the ground lead for all biopotential sensors attached to the right pelvis, and the reference lead on the right collarbone. All biopotential sensors used standard clinical adhesive $\mathrm{Ag} / \mathrm{AgCl}$ snap-on electrodes (3M, Inc.). We then positioned the strip-imaging sensor (Fig. 2, right) on the upper arm wrapping around from the lateral head of the triceps brachii around anteriorly to the biceps brachii. The accelerometer was positioned on the back of the subject's hand, with the force sensor positioned on her fingertips and temperature sensor under her arm. The subject performed isometric flexions alternately lifting up or pressing down on a table at arm's-length for $15 \mathrm{~s}$. Force production was alternated with $30 \mathrm{~s}$ of rest, with three full cycles. The goal was to demonstrate high frame rate $(25-\mathrm{Hz})$ tomographic imaging and video of muscle hemodynamics during the task.

Multimuscle monitoring during stationary cycling: One healthy subject (female, age 33) was recruited for this test. The ground lead (for all biopotential sensors) was attached at the right pelvis and the reference lead at the right clavicle. 
Three ECG leads were attached at positions V2, V4, and V6. Four EMG leads were attached at the inferior aspects of rectus femoris and vastus lateralis in the upper right leg and the tibialis anterior and gastrocnemius lateralis (GL) of the lower right leg. Four NIRS sensor pads [Fig. 2(b)] were placed over central aspects of the same four muscles. An accelerometer sensor was attached on the lateral aspect of the right knee, a force sensor under the ball of the right foot, and a temperature sensor on the right torso, under the arm. The subject was seated on a stationary electromagnetically braked cycle ergometer and performed intermittent 20 -s bursts of vigorous pedaling at (120 rpm at $100 \mathrm{~W}$ ), which were alternated with $20 \mathrm{~s}$ of rest. The subject completed four full cycles of the activity.

Muscle monitoring while running: Figure 3 illustrates how NINscan-M can be deployed during running. For this test, three ECG leads were attached at positions V2, V4, and V6, and four EMG leads were attached at the inferior aspects of vastus intermedius, vastus intermedius inferior, vastus lateralis, and biceps femoris of the right leg. The ground lead for all biopotential sensors was again attached at the right pelvis and the reference lead at the right clavicle. Our aDOT strip-imaging sensor was wrapped from the head of biceps femoris, anteriorly over vastus lateralis and to the iliotibialis tract. Accelerometer and temperature sensors were attached on the inferior aspect of the vastus intermedius under the NIRS probe. The force sensor was attached under the ball of the right foot. All sensors were secured to the subject's torso and leg by athletic tape. The data were collected outside on a sidewalk. The subject performed intermittent 20 -s sprints alternated with $20 \mathrm{~s}$ of walking at a comfortable pace, and the subject completed eight full cycles of the activity.

Biopotential measurement capabilities: We also conducted two tests designed to illustrate the auxiliary capabilities of the system. First, we connected seven leads (plus reference and ground) per standard multilead clinical ECG monitoring protocols. ${ }^{49}$ Recording was conducted for $30 \mathrm{~s}$ to demonstrate the signal characteristics of NINscan-M using different biopotential gain settings. To test the sensitivity of signals much smaller than EMG or ECG, we then positioned a biopotential sensor on the subject's forehead (location F3 per the international

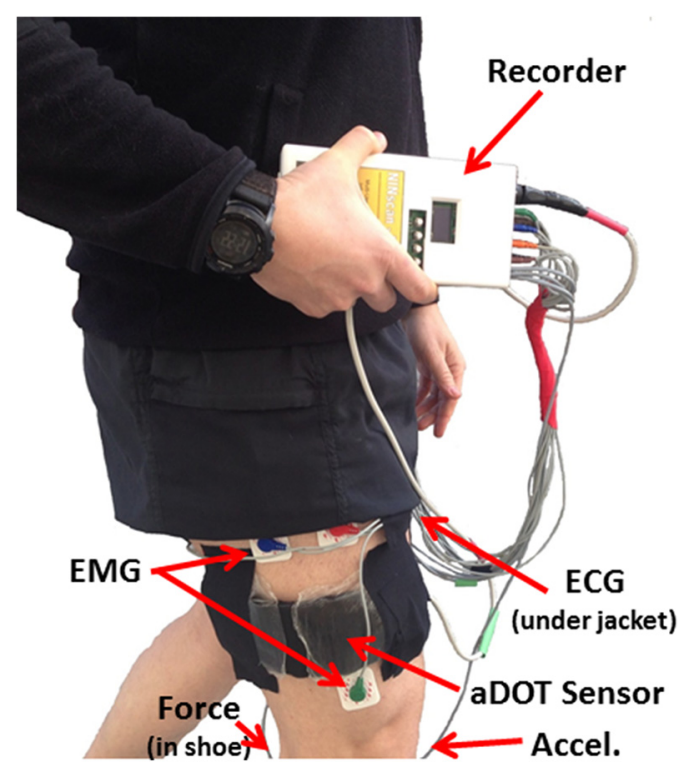

Fig. 3 Configuration of NINscan-M setup during free-running.
10/20 system) and recorded EEG while the subject performed seven cycles of the following protocol: $5 \mathrm{~s}$ of quiet rest, $10 \mathrm{~s}$ of circular eye-rolling, $10 \mathrm{~s}$ of rapid blinking, $20 \mathrm{~s}$ of rest with eyes open, and $20 \mathrm{~s}$ of rest with eyes closed. These tasks were selected to generate low-frequency EEG artifacts (eye movements), a broad-spectrum low-amplitude EEG (eyes-open rest), and enhanced activity in the alpha band (7 to $15 \mathrm{~Hz}$ ) during eyes closed. ${ }^{50}$

\subsection{Data Analysis}

Being a continuous-wave instrument, NINscan-M can only measure relative changes in oxy-/deoxy-Hb concentrations. The raw 250-Hz optical "difference" data (raw-background) were first digitally low-pass filtered at $5 \mathrm{~Hz}$ followed by application of the modified Beer-Lambert $\mathrm{Law}^{51}$ and our previous approach, ${ }^{23}$ or the DOT imaging process described as follows. For the 250-Hz ECG data, we plot the raw data after notch-filtering at 60 and $120 \mathrm{~Hz}$ (second-order Butterworth bandstop filters) to reject any power line noise. For the 250-Hz EEG data, we similarly notch-filtered at 60 and $120 \mathrm{~Hz}$, and then computed the spectrogram using a Hanning filter of $1 \mathrm{~s}$ and window-overlaps of $0.5 \mathrm{~s}$.

\subsection{Diffuse Optical Tomography Imaging Procedure}

With data collected from the 64 NIRS channels, 3-D dynamic diffuse optical imaging was performed using a linear image reconstruction algorithm, due to its low calculation load and fast imaging speed. ${ }^{52,53}$ The algorithm has been thoroughly tested on phantoms ${ }^{54}$ and previously validated for breast imaging. ${ }^{55}$ In brief, the diffusion approximation to the radiative transport equation and analytical solution based on Rytov approximation and semi-infinite boundary condition were used for forward modeling. Since the inversion of the linear system derived from the forward modeling is both ill-posed and underdetermined, regularization or stabilization techniques are required to obtain physically meaningful results. Here, we used truncated singular value decomposition, a subspace technique. In our imaging procedure, only reduced absorption coefficients were reconstructed; scattering was assumed to be unchanged during the test. The tissue beneath the DOT probe was divided into 5-mm-thick layers with $10 \times 10 \mathrm{~mm}$ in-plane resolution, and image reconstruction was performed in MATLAB using the photon migration imaging toolbox. ${ }^{56}$ To enhance the contrast and reduce image artifacts, the reconstructed images were smoothed using a $3 \times 3 \times 3$ voxel median filter.

\section{Performance Tests}

\subsection{Instrument Characterization}

The detailed specifications of NINscan-M are shown in Tables 1 and 2. NINscan-M supports high-density SD memory cards (e.g., 32 GB). Continuous data collection of all NIRS, analog and digital channels generates a data flow of up to $147 \mathrm{MB} / \mathrm{h}$, enabling NINscan-M to support long-term continuous data collection for more than a week when run off wall power. With four regular AA sized Ni-MH rechargeable batteries (2500 mAh), NINscan-M can perform continuous data collection for up to $26 \mathrm{~h}$. Since NINscan-M (i) supports a large memory, (ii) can collect data without a computer, and (iii) functions without distance restrictions (as those from wireless ambulatory NIRS devices), it is a fully independent and stand-alone 
Table 1 Basic specifications of NINscan-M.

\begin{tabular}{ll} 
Dimensions & $95 \mathrm{~mm} \times 180 \mathrm{~mm} \times 38 \mathrm{~mm}$ \\
Weight & $577 \mathrm{~g}$ (including batteries) \\
$\begin{array}{l}\text { Working current/power } \\
\text { consumption }\end{array}$ & $150 \mathrm{~mA} / 0.72 \mathrm{~W}$ \\
Sampling rate & Up to $250 \mathrm{~Hz}$ \\
A/D converter resolution & NIRS: 16 bits \\
& Biopotential: 24 bits \\
& Force/respiration: 12 bits \\
$\begin{array}{ll}\text { Onboard memory } & \text { SD card (typically } 32 \mathrm{~Gb}, \\
\text { or }>215 \mathrm{~h} \text { of data) }\end{array}$ \\
$\begin{array}{l}\text { Number of sensor channels } \\
\text { (typical) }\end{array}$ & 47 digital, 1 analog) \\
$\begin{array}{l}\text { Battery life (four AA size, } \\
\text { rechargeable) }\end{array}$ & $26 \mathrm{~h}$ \\
$\begin{array}{l}\text { Frequency range of NIRS channels } \\
\text { Frequency range of biopotentials }\end{array}$ & 0 to $10 \mathrm{~Hz}$ \\
$\begin{array}{l}\text { Frequency range of force/respiration } \\
\text { re } 70 \mathrm{~Hz}\end{array}$ & 0 to $5 \mathrm{~Hz}$ \\
\hline
\end{tabular}

device. This system is thus suitable for the widest range of application environments, including extreme conditions such as spaceflight, mountaineering, and long-distance running.

The bandwidth of each channel is designed according to the characteristics of each parameter. For example, analog auxiliary inputs have a 70-Hz bandwidth, commonly used in ambulatory ECG, whereas the NIRS channels have a 5-Hz bandwidth. Further signal conditioning can be performed via analysis after data collection.

Table 2 summarizes the performance characteristics of the optical channels, the core of aDOT monitoring. For battery powered long-term ambulatory monitoring, signal drift is a critical parameter, since the voltage of the battery decreases as its power is consumed and it is important that the signal channels are resistant to the battery voltage change. Our test results found the drift over a $24-\mathrm{h}$ period is $<1 \%$, excellent for long-term

Table 2 Basic characterization of NINscan-M's NIRS channels.

NEP

Instrument dynamic range

Drift (stability)

Linearity error

Cross talk

Optical output power

Wavelengths
$15 \mathrm{pW} / \sqrt{\mathrm{Hz}}$

$180 \mathrm{~dB}$

$1 \%$ per $24 \mathrm{~h}$

$<5 \%$

$-60 \mathrm{~dB}(0.1 \%)$

0 to $10 \mathrm{~mW}$

780 and $830 \mathrm{~nm}$ monitoring applications. The NEP of NINscan-M was found to be $15 \mathrm{pW} / \sqrt{\mathrm{Hz}}$, including both the dark noise from the OPT101 detector $(\sim 5 \mathrm{pW} / \sqrt{\mathrm{Hz}})$ and the noise from the circuitry that follows. The $180-\mathrm{dB}$ dynamic range includes the instantaneous 70-dB dynamic range of the detector itself, plus an extra $110 \mathrm{~dB}$ from the variable gains. These characteristics compare favorably with many existing nonportable units.

\subsection{Isometric Muscle Oxygenation Time Series Imaging}

A full set of synchronized NINscan-M sensor data from the isometric contraction experiment is shown in Fig. 4. Time series data for deoxy-Hb concentrations from the 23 NIRS sourcedetector pairs with signal-to-noise ratios (SNR) $>3$ during the isometric biceps/triceps activation task appear in Fig. 4(a). (Arbitrary offsets on the $y$-axis are added for individual trace visibility.) These include source-detector separations ranging from 12 to $56 \mathrm{~mm}$ [see labels in Fig. 2(c)]. Biceps deoxygenation was largest during the biceps flexion periods (and tended to reach a plateau), although it was still detectable during the triceps activation periods (coactivation). Triceps deoxygenation was most pronounced during triceps flexion, did not reach a plateau, and changes were also observed during biceps flexion periods. The accelerometer (hand motion), force sensor data, and temperature data for the same recording appear in Fig. 4(b). From these, we can verify limited motion of the hand during the (isometric) task and quantify the forces generated on the sensor. The deployed digital temperature sensor is relatively of low resolution $\left(0.25^{\circ} \mathrm{C}\right)$ but demonstrates stable body temperature during the task. The synchronously recorded EMG and ECG recordings appear in Fig. 4(c), showing clear bursts of muscle activation during the contraction periods and quiescent signals during rest. Importantly, coactivation of biceps and triceps was observed, which guides interpretation of the oxygenation data above. Additional noise is noted in the ECG traces during the muscle activation periods.

We then combined the probe geometry from Fig. 2(c) and the data from Fig. 4 with our tomographic imaging algorithm as described in Sec. 2.9. Given the probe dimensions, 3-D images were reconstructed over a space of $X=0$ to $11 \mathrm{~cm}, Y=0$ to $4 \mathrm{~cm}$, and $Z=0$ to $2 \mathrm{~cm}$ in depth, with voxel sizes of $1 \times 1 \times$ $0.5 \mathrm{~cm}$, or a total of 220 voxels.

Using this geometry, we generated separate images of oxy-, deoxy-, and total-hemoglobin [Fig. 5(a)], as well as images (and videos at $25-\mathrm{Hz}$ frame rates) of each parameter in depth [Fig. 5(b)]. The video illustrates spatial details regarding the deoxygenation and associated postrelease hyperemia phases of the task, including an apparent involvement of the region of the brachialis and biceps (in this example subject) during the recovery hyperemia for triceps activation.

\subsection{Muscle Activation Monitoring During Stationary Cycling}

In our second test, we simultaneously examined muscle oxygenation in four separate muscles in the upper and lower leg using our quad probe [Fig. 2(b)] during repeated 20-s bursts of high-rpm pedaling on an electromagnetically braked cycle ergometer (Corival 912900, Lode BV, Netherlands). Data from two such bursts appear in Fig. 6, showing the higher initial force required to start cycling, the lower maintenance forces, and the rate of motion/acceleration of the knee (which is out of phase 

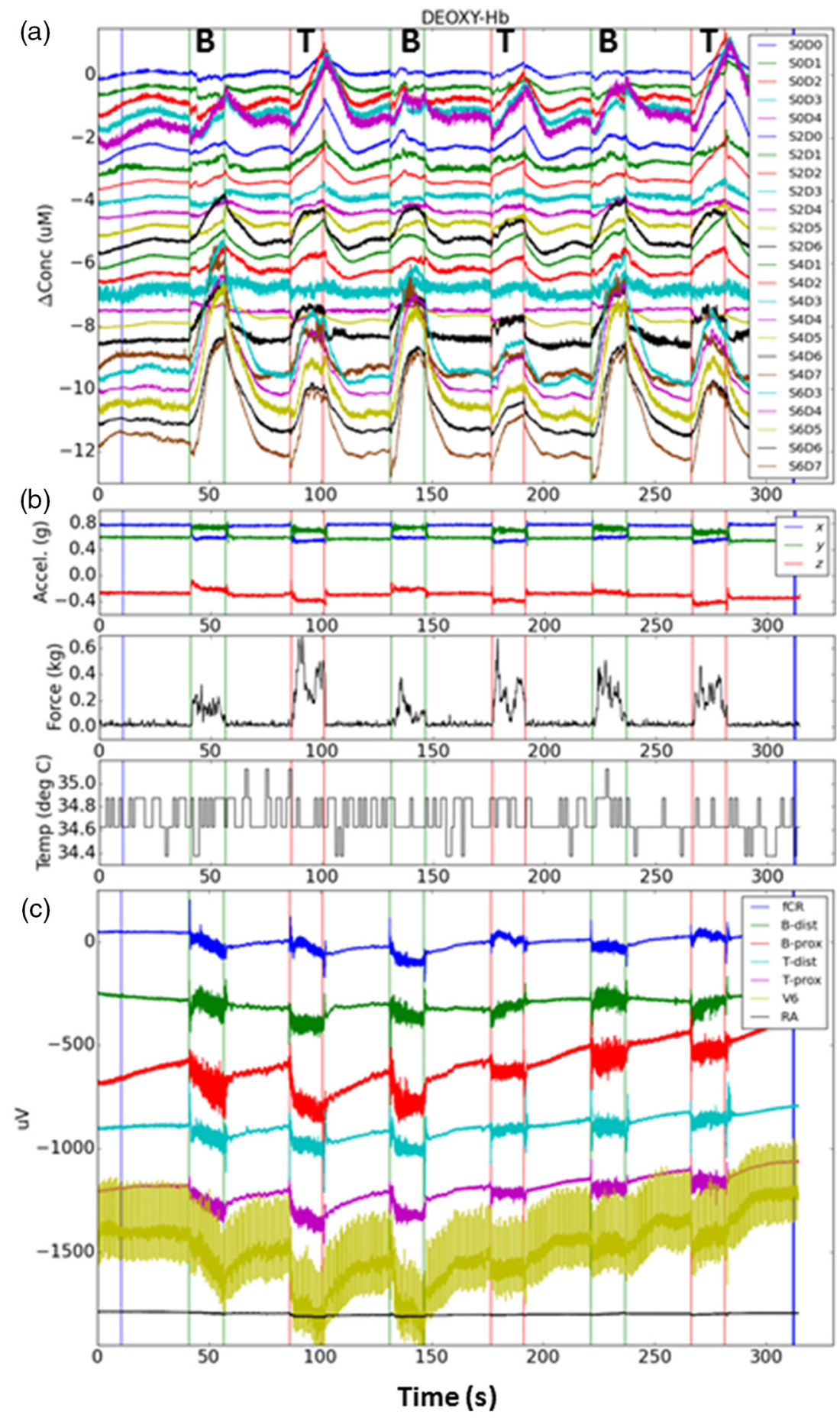

Fig. 4 (a) Deoxy-Hb concentration changes over time during periods of biceps flexion (columns labeled $B$ ), triceps flexion (columns labeled $\mathrm{T}$ ), and rest (unlabeled). The legend refers to sources and detectors in the strip-imaging sensor depicted in Fig. 2(c). (b) Synchronously recorded accelerometry measured from the back of the hand (top), force generated (middle), and temperature sensor output (bottom) during isometric flexions. (c) Synchronously recorded EMG from the body of the fCR, distal biceps brachii (B-dist), proximal biceps (B-prox), distal triceps, proximal triceps, and ECG (location V6), plus the reference electrode during the isometric flexion task.

with the force data). Increases in deoxy-Hb were associated with cycling bursts in the GL. The tibialis anterior exhibited minimal change in deoxy-Hb (or even a modest decrease during cycling bouts) for this muscle opposing the GL. The altered deoxy-Hb concentrations returned toward baseline following each cycling bout.

\subsection{Ambulatory Muscle Oxygenation Imaging}

Our third test involved aDOT in a freely running subject. Figure 7(a) illustrates the setup and the positioning of the sensor relative to the underlying musculature. Figures $7(\mathrm{~b})$ and $7(\mathrm{c})$ show oxy- and deoxy-Hb concentration changes collected 

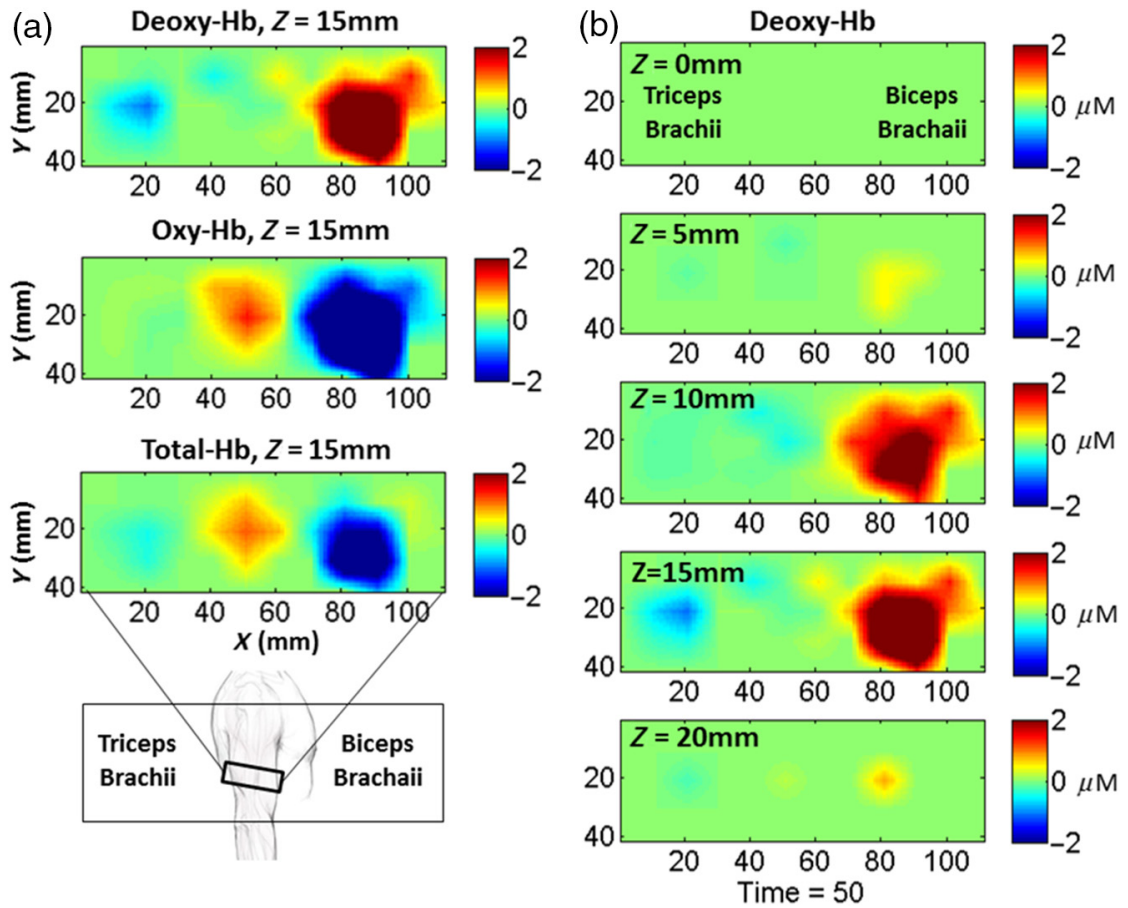

Fig. 5 (a) DOT images of deoxy-, oxy-, and total-Hb changes from resting baseline at 15-mm depth during peak biceps flexion, with the right end of the probe/image overlying biceps brachii. Inset indicates approximate NIRS sensor position and orientation. (b) Deoxy-Hb time-series imaging in depth (0 to $20 \mathrm{~mm}$ ), with real-time video at $25 \mathrm{~Hz}$. Still image comes from the same time point (50 s) as imaged in (a). (Video 1; MPEG, 10 MB [URL: http://dx.doi.org/10.1117/1.JBO.21.9.091314.1]).

from the lateral thigh during the 20 -s active-running period compared to the initial walking period. The auxiliary measures further allowed us to quantify and synchronize individual footfalls (accelerometer), muscle electrical activation (EMG), and cardiac information (ECG), and skin temperature along with the aDOT data (not shown).

\subsection{Electrophysiological Biopotential Recordings}

To claim multimodality capabilities suitable for muscle and exercise monitoring requires high-quality electrophysiological inputs. In Fig. 8(a), we show data using our NINscan-M hardware platform for ECG-array recording, as would be deployed with cardiac or cardiovascular patients undergoing exercise or muscle testing. All signals were very low noise $(<2 \mu \mathrm{V})$, and data with reasonable (if suboptimal) SNRs were obtained even at the lowest gain setting available.

ECG and EMG are generally high-amplitude signals, and hence more straightforward to detect. EEG recording provides a much more challenging test, while at the same time being important to the investigation of muscle fatigue. ${ }^{57,58}$ From our frontal EEG test, Fig. 8(b) shows high-amplitude low-frequency components (dark red), which were largely confined to the eye movement periods of the protocol, representing eye-movement artifacts in the EEG signal. In addition, lighter red bands consistently appear in the $11-$ to $13-\mathrm{Hz}$ range during the final $20 \mathrm{~s}$ of each cycle (arrows). These bands correspond to the common increase in alpha-wave power during eyes-closed rest periods $s^{50}$ - typically most prominent in the occipital lobe, but sometimes observable frontally as well. While limited, this test provides evidence that, in addition to sensitivity to ECG in the order of $500 \mu \mathrm{V}$, the NINscan-M hardware platform is also sensitive to the much smaller signals from EEG, in the order of 1 to $10 \mu \mathrm{V}$.

\section{Discussion}

We have described here NINscan-M, a system capable of 64-channel mobile NIRS tomographic imaging of muscle or other tissue hemodynamics along with simultaneous, multimodal physiological measurements including ECG, EMG, EOG, EEG, accelerometry, force, and temperature. Versions of this device have been further enhanced to incorporate gyroscope and respirometry sensors. The system design provides a NIRS dynamic range of up to $180 \mathrm{~dB}$, and we could reliably acquire NIRS imaging data with source-detector separations ranging from 12 to $56 \mathrm{~mm}$. Based on prior studies on forearm ${ }^{59}$ and head, ${ }^{44,60}$ measurements with such source-detector spacings are expected to be sensitive to deep tissues such as muscle and brain. Given the automatic and dynamic gain control, NINscan-M can also support multisite tissue monitoring by simply reconfiguring sources and detectors into separate pads [e. g., Fig. 2(b)]. This design provides a significant advantage over many NIRS approaches, including various nonportable ones, as it enables 3-D tomographic imaging (as opposed to 2-D topographic imaging), as well as nearly arbitrary source-detector geometries for different coverage and shallow/deep layer measurement needs. ${ }^{42,61}$ The system portability enables imaging/ monitoring of muscle oxygenation changes in static, dynamic, and freely-moving exercise activities. And the multimodal physiological recording capabilities can help provide a more comprehensive understanding of muscle physiology and in a wide variety of testing environments. ${ }^{16}$ 


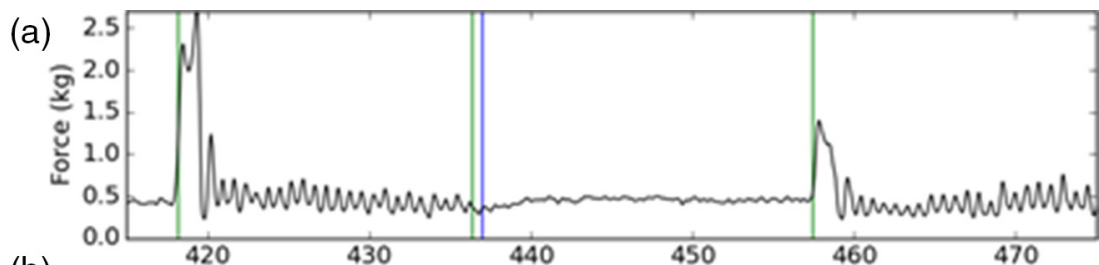

(b)

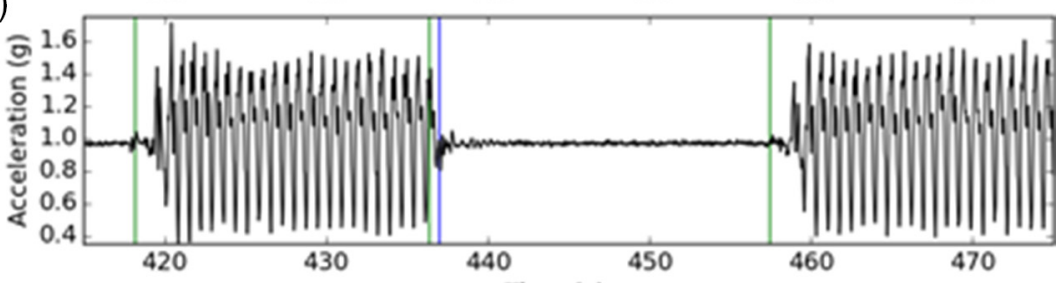

(c)
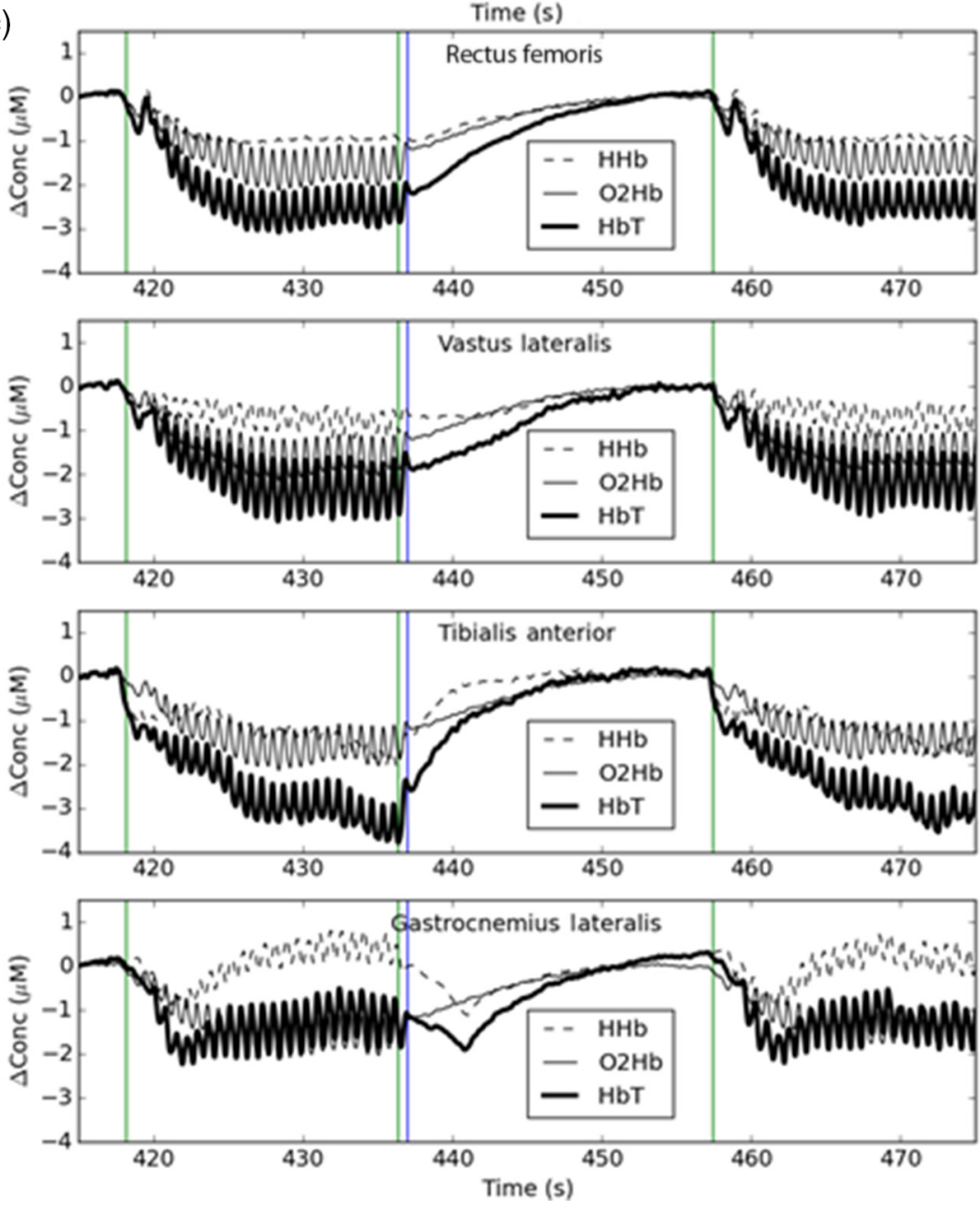

Fig. 6 Two 20-s bursts of rapid cycling ( $100 \mathrm{~W}$ at $120 \mathrm{rpm}$ ), plotted at the full sample rates of the recorded data $(250 \mathrm{~Hz}$ for acceleration, $25 \mathrm{~Hz}$ for force and NIRS). (a) Force generated on the right ergometer pedal, (b) total acceleration on the lateral aspect of the right knee, (c) NIRS-recorded deoxy-Hb concentration changes over the (top to bottom): $R$ rectus femoris (SOD1), $R$ vastus lateralis (S2D3), $R$ tibialis anterior (S4D5), and R GL (S6D7).

\subsection{Technology Considerations}

Despite NINscan-M's fourfold increase in supported sources and detectors, our NIRS probe design was substantially similar to prior work. ${ }^{23}$ In particular, we used a lightweight, Velcrobased sandwich form to hold the laser diodes and photodiodes. A medical tape layer is used for biocompatibility, and light shaping diffusers are attached to the surface of the laser diodes to reduce the effective laser class to class 1 (eye-safe). All other sensor-patient interfaces deployed standard clinical electrodes. This provides a lightweight, low-profile, and biocompatible interface with human subjects.

Several new design challenges arose relative to our previous device development efforts. First, a mobile tomography system proved challenging in terms of power consumption, since more lasers draw more current. This problem was solved via TDM 

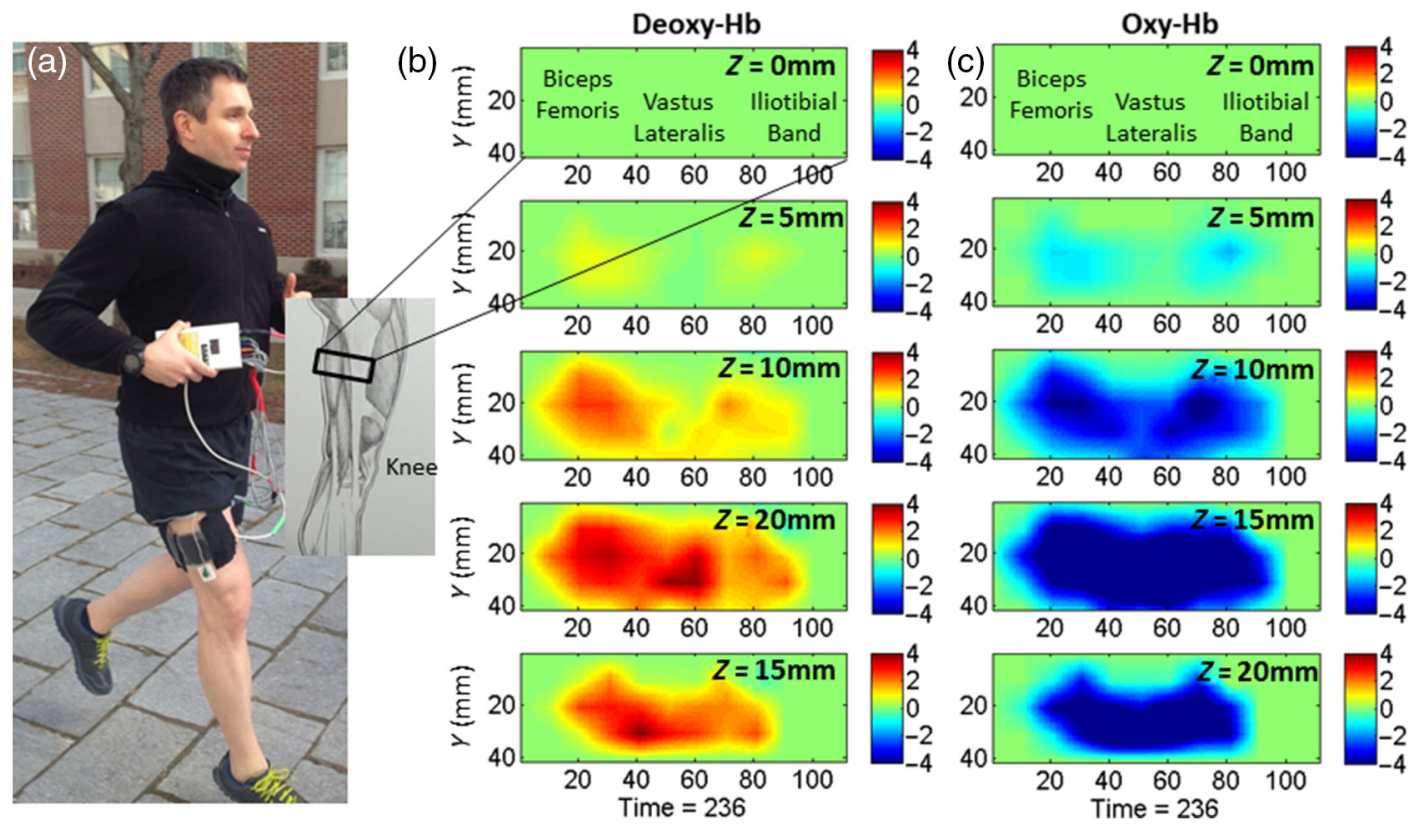

Fig. 7 (a) Photo of NINscan-M during free running, along with illustration of the location of the NIRS imaging sensor (rectangle). ( $b$ and c) DOT images of deoxy- $\mathrm{Hb}$ and oxy-Hb concentration changes in depth $(0$ to $20 \mathrm{~mm})$ during active running relative to walking. Less activation at the right end of the image reflects that part of the probe being located over the iliotibial tract.
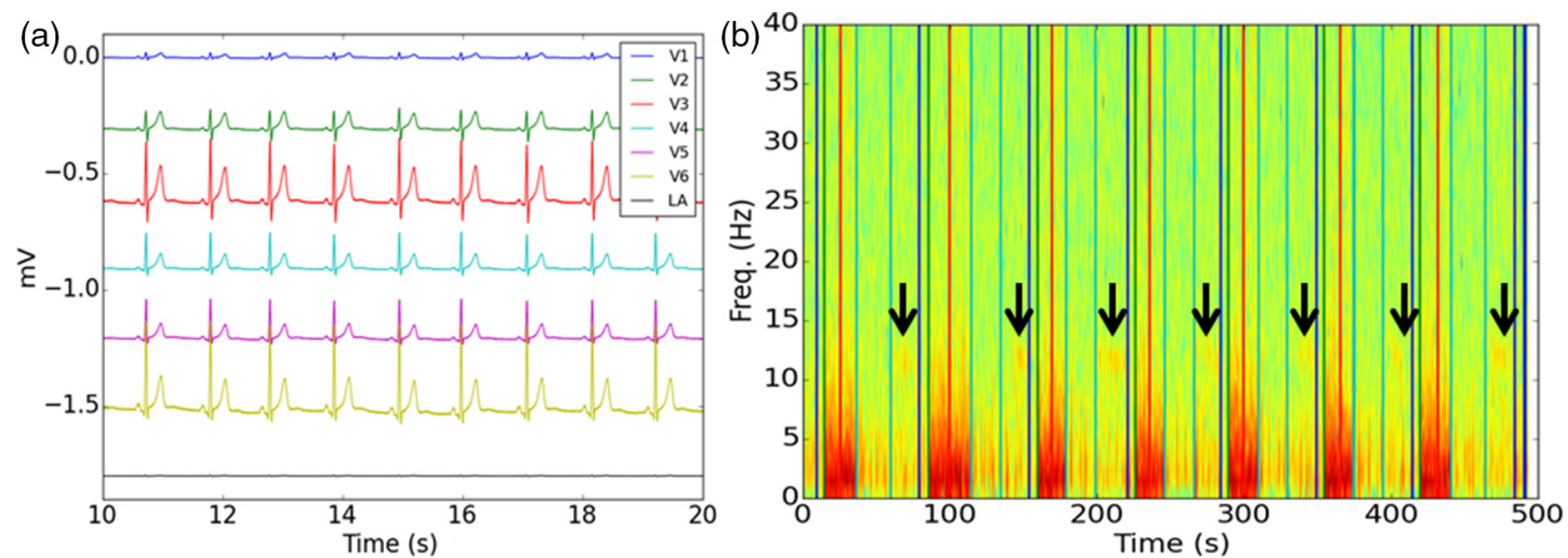

Fig. 8 (a) NINscan-M recording from a 9-lead ECG array (including reference and ground leads) at multiple gain settings $(2 \times, 4 \times, 12 \times, 6 \times, 8 \times$, and $24 \times$ for $V 1$ to $V 6$, top to bottom, respectively). (b) EEG at F3 during seven cycles of: 5 -s rest, 10-s eye rolling, 10-s eye blinking, 20-s quiet eyes-open rest, and 20-s eyes-closed rest. This illustrates the low noise associated with the electrophysiological recording component of NINscan-M.

and real-time nulling of ambient light levels. This solution also enabled incorporation of real-time gain control, which significantly expanded the prototype's dynamic range. However, TDM slows the device frame rate, and slower speeds can be inadequate for capturing important physiology or fail to reject sudden changes in ambient lighting. The slowest link proved to be the dynamic gain switching within the probe itself, especially notable at low signal levels. For this reason, two system modes were included: (i) a $250-\mathrm{Hz}$ frame rate when setting the on-probe gains at power-up only, keeping them fixed during recording or (ii) a $25-\mathrm{Hz}$ frame rate when enabling dynamic on-probe gain changes. Since slow gain-switching only affects the NIRS subsystem, biopotential and other sensors are sampled at $250 \mathrm{~Hz}$.
As with any system, NINscan-M has limitations. One important limitation is that the device can currently output a maximum of $10 \mathrm{~mW}$ of peak optical power. With coupled optics and the TDM design, the net optical power can be considerably below the American National Standards Institute (ANSI) limit for skin exposure to near-infrared light. Higher light levels are particularly important for photodiodes such as OPT101, which are less sensitive to low light levels than avalanche photodiodes or photomultipliers. In addition, our low-power device requirement means we supply only $2.5 \mathrm{~V}$ to the detector, thus underutilizing the capabilities of OPT101. While we were able to achieve robust signals with source-detector separations $>55 \mathrm{~mm}$, the limits on light power and on the OPT101 supply voltage limit 
the SNR and, consequently, the depth sensitivity of the system. A higher-output power board could mitigate this limitation but remains to be developed.

We also observed baseline fluctuations and cross talk between biopotential channels. Baseline fluctuations are a limitation of the ASD1299 chip and can be partially managed by incorporating software or hardware filters as needed. Despite our varied experimental conditions, we have not encountered signal saturation due to baseline fluctuations, even when using a $24 \times$ gain on the biopotential channels, and hence this has been of limited concern. Cross talk is more challenging and has three sources: (i) cross talk within ADS1299 (a known issue), as well as (ii) physiological, and (iii) referencing cross talk. Within-chip cross talk is particularly likely, and difficult to avoid, if one must simultaneously record large signals such as ECG with small signals such as EEG. There is also physiological coupling between sensor sites on the body during muscle activity, leading to inherently more noise during movement than when remaining still, and this generally inherent to the subject. Regarding the third potential source of cross talk, our tests used a single reference channel for all EMG, ECG, and EEG measurements and plotted raw data. Rereferencing (e.g., standard ECG or EEG montages) or deploying separate reference leads to the different types of measurements (EEG versus ECG versus EMG, and so on), which are both expected to help reduce cross talk in the electrophysiological signals, and may reduce the effect of physiological cross talk as well. Postprocessing filters may also be required to help eliminate some types of cross talk, however, depending on the particular application.

Another important consideration is line-noise. Although it is generally run on batteries, NINscan-M is also capable of plug-in operation and will regardless be susceptible to $50-$ or $60-\mathrm{Hz}$ line noise coupling. Because the NIRS component of NINscan-M often runs at $25 \mathrm{~Hz}$, any such line noise would be aliased. When running at $250 \mathrm{~Hz}$, we have not observed significant $60-\mathrm{Hz}$ interference in the NIRS channels, whereas we did see such noise in the $250-\mathrm{Hz}$ biopotential subsystem when not suitably grounded or shielded. Nevertheless, line noise in the NIRS signals remains a potential concern. One possible solution is to run the system at $125 \mathrm{~Hz}$. This should be slow enough to minimize problems regarding the on-probe gain switching speed, yet fast enough to avoid line noise aliasing.

As with any ambulatory instrument, NINscan-M will be sensitive to motion artifacts. Numerous solutions to this problem have been proposed, including development of lightweight, motion-resistant sensors ${ }^{23}$ as well as various filtering approaches. ${ }^{41,62-64}$ We have approached this in a similar manner to our previous work, ${ }^{23}$ minimizing the NIRS probe profile and mass via use of soft, lightweight materials, elimination of weighty and torque-inducing optical fibers and, when needed, attachment of 3-mm diameter lenses directly to the face of the detectors (using optically transparent glue) to improve performance in hairy regions.

\subsection{Imaging Considerations}

Measurement noise is always a concern, but particularly so for NIRS data that must support tomographic imaging. We found acceptable signals $(\mathrm{SNR}>3$ ) could be reliably achieved up to at least 56-mm source-detector separations and meaningful aDOT images could be obtained up to $20 \mathrm{~mm}$ in depth. Imaging was based on algorithms previously used and thoroughly validated for breast imaging applications. ${ }^{55}$ While we reconstructed images with $10 \times 10 \mathrm{~mm}$ in-plane resolution and smoothed the result, the theoretical and practical spatial resolution remains to be fully characterized. We expect imaging from NINscan-M to be equivalent to that of other systems with similar sensitivity, although more comprehensive imaging validation remains to be completed for muscle applications, including imaging based on cylindrical boundary conditions. ${ }^{65}$

\subsection{Spaceflight Muscle Applications}

For spaceflight, two critical features for all biomedical devices are multiuse and ease-of-use. The multiuse requirement led to the multimodal recording capabilities in NINscan-M. For example, in a spaceflight analog study (supine cycling), DiMenna et al. $^{38}$ combined EMG and force data with oxygenation data collected during muscle activation paradigms to assess muscle strength and muscle endurance. Their 15-min endurance assessment could replace a $2+\mathrm{h}$ time trial, which is an important time savings benefit in spaceflight settings. However, DiMenna's study required multiple laboratory devices, whereas similar studies could be conducted exclusively with NINscan-M. Other works have further demonstrated the possibility of optimizing both endurance and strength training protocols when combining NIRS with various auxiliary measures. ${ }^{11,38}$ NINscan-M thus has the potential to provide a single, small device suitable for monitoring changes in muscle strength and endurance as well as optimizing exercise protocols during long-duration spaceflight. The spaceflight ease-of-use requirement led to the current design whereby the user only needs to position the sensors on the body as desired and switch the device on/off as needed. Gain settings, data recording, and file storage are all handled automatically, which can save valuable time in high-tempo operational settings.

\subsection{Earth Muscle Applications}

The data collected in this study was designed to highlight the system's range of applications and multimodal capabilities, as opposed to testing specific physiological hypotheses, and our results were broadly consistent with previously published work. ${ }^{16,22,40,66}$ In particular, the isometric studies replicated prior demonstrations of a fast initial deoxygenation followed by a slow phase during biceps brachii flexion, with similar amplitudes of $[\mathrm{HHb}]$ change. ${ }^{66}$ Simultaneous EMG recordings help explain the bilateral NIRS deoxygenations by demonstrating co-contraction of opposing muscle groups during the task. The rapid $(>1 \mathrm{~Hz})$ fluctuations during cycling and running (Fig. 6) are consistent with prior NIRS studies of active motion, e.g., 16,66,67 and are most likely related to sensor coupling and/or tissue deformation beneath the sensor. The underlying, more prolonged deoxygenation observed during blocks of muscle activation are also consistent with these prior studies in terms of amplitude and timescale, ${ }^{66,67}$ and represent underlying changes in muscle utilization and oxygen extraction. Baseline muscle $\mathrm{Hb}$ concentrations are typically $\sim 80 \mu \mathrm{M}^{27}$ Thus, our observed oxy-/deoxy-Hb concentration changes in all of the tasks (ranging up to $\sim 4 \mu \mathrm{M}$ ) represent $\sim 5 \%$ a change from baseline. While these magnitudes are similar to prior reports, amplitude discrepancies between studies can reach up to 10-fold. ${ }^{22,66}$ Likely contributors to such differences include variations in human performance or experimental controls, spatial variations within the muscle, ${ }^{31,68}$ different approaches for handling the skin 
and fat layers, ${ }^{69}$ and perhaps simple interindividual variability as previously reported. ${ }^{22,70}$ Further studies are needed to better understand the source of such variations.

Our findings nevertheless suggest that NINscan-M can provide tissue oxygenation imaging data during active exercise, and hence is applicable to studies of muscle strength and endurance, fatigue, and, potentially, training optimization studies. In addition to the muscle strength and endurance assessments described above, a wearable system can support detailed exercise physiology studies in more realistic settings, enabling quantification of tissue oxygen use, force production, muscle activation, cardiac and respiratory variables while running, cycling, skiing, or other participation in activities on various training or competition courses. As an example, studies have incorporated EEG in conjunction with force and muscle oxygenation data to investigate the nature of fatigue. ${ }^{57,58}$ NIRS has also been combined with electrophysiological measures for investigating or managing various clinical conditions that would not typically be encountered in spaceflight including peripheral vessel diseases, ${ }^{71}$ congestive heart failure, ${ }^{72}$ compartment syndrome, ${ }^{73}$ and diabetes. ${ }^{74}$ This system could help support similar investigations in fully ambulatory settings.

The range of potential sensors in NINscan-M means the system could be deployed for various nonmuscle applications on Earth as well. For example, when using the respiration sensor, the system could be used for basic polysomnography in low-resource settings. Alternatively, skin conductance sensors could be incorporated to record multimodal physiological responses to stress ${ }^{75}$ or blood pressure sensors could help perform symptom-capture investigations of syncope. ${ }^{76}$ The key feature of NINscan-M is that it is a fully ambulatory physiological monitoring platform, which can help provide a more comprehensive picture of physiological state during vigorous, remote, or ambulatory activities.

While we have focused on muscle applications here, the NIRS subsystem of NINscan-M was also designed to enable aDOT of brain activity. The same device is thus expected to have applications for remote, long-duration and/or ambulatory cerebral monitoring. Applications in spaceflight would include cerebrovascular pulsatility, ${ }^{77}$ cerebral autoregulation, ${ }^{78}$ or changes in functional brain activation under stress, heavy workload, or following specific events. ${ }^{79-81}$ Earth-based applications, where NINscan-M provides a unique capability, include detection and diagnosis of various disorders characterized by intermittent or unexpected cardiac symptoms (e.g., arrhythmias, transient ischemic episodes, and silent myocardial ischemia), ${ }^{1}$ as well as syncope,${ }^{82}$ epilepsy, ${ }^{83}$ poststroke monitoring, ${ }^{84}$ or sleep disorders. $^{85}$

\subsection{Summary}

Our overall goal has been to develop a highly portable and multiuse system for tomographic imaging in muscle and brain plus systemic physiological monitoring suitable for use by astronauts during spaceflight. We have also sought to enable a range of clinical and research applications outside the confines of a hospital or laboratory. NINscan-M represents a significant step along this path, coupling a highly portable and flexible aDOT capability with low-power consumption and motion resistance to multimodal recording capabilities and ease-of-use. It can address important gaps in multimodal monitoring and enables arbitrary close-detector measurements to support skin and fat layer interference correction, all in a self-contained device. For muscle and exercise applications, NINscan-M's capabilities could support muscle strength, endurance, recovery, and fatigue assessment, guidance on training paradigms, or clinical conditions, such as compartment syndrome. Sensitivity to source-detector separations exceeding $\sim 25 \mathrm{~mm}$ expands its potential applications to cerebral applications, ${ }^{43,44}$ potentially enabling the detection and assessment of hemorrhage, longduration postsurgical monitoring in the ICU, traumatic brain injury applications, and even stroke, epilepsy, or syncope monitoring. This general hardware platform thus enables research and clinical applications involving muscle, as well as applications in brain and more systemic physiological monitoring and imaging.

\section{Acknowledgments}

This work was supported by the National Space Biomedical Research Institute through NASA NCC 9-58.

\section{References}

1. N. J. Holter, "The development of Holter electrocardiography," Clin. Eng. 8(6), 65-67 (1980).

2. A. Shoeb et al., "Patient-specific seizure onset detection," Epilepsy Behav. 5(4), 483-498 (2004).

3. A. Shoeb et al., "Detecting seizure onset in the ambulatory setting: demonstrating feasibility," in 2005 IEEE Engineering in Medicine and Biology 27th Annual Conf., Vol. 4, pp. 3546-3550 (2005).

4. A. Covic, A. A. Haydar, and D. Goldsmith, "Ambulatory blood pressure monitoring in hemodialysis patients: a critique and literature review," Semin. Dial. 17(4), 255-259 (2004).

5. C. W. Barratt et al., "Selection of pulse oximetry equipment for ambulatory monitoring," J. Med. Eng. Technol. 25(1), 17-24 (2001).

6. C. Worringham, A. Rojek, and I. Stewart, "Development and feasibility of a smartphone, ECG and GPS based system for remotely monitoring exercise in cardiac rehabilitation," PLoS One 6(2), e14669 (2011).

7. K. E. Iscoe et al., "Efficacy of continuous real-time blood glucose monitoring during and after prolonged high-intensity cycling exercise: spinning with a continuous glucose monitoring system," Diabetes Technol. Ther. 8(6), 627-635 (2006).

8. W. N. Colier et al., "Determination of oxygen consumption in muscle during exercise using near infrared spectroscopy," Acta Anaesthesiol. Scand. Suppl. 39, 151-155 (1995).

9. R. A. De Blasi et al., "Cerebral and muscle oxygen saturation measurement by frequency-domain near-infra-red spectrometer," Med. Biol. Eng. Comput. 33(2), 228-230 (1995).

10. M. Ladewig, R. Robertson, and E. M. Nemoto, "Muscle oxygenation by near infrared spectroscopy and lactate thresholds in endurance trained and recreationally active cyclists," Adv. Exp. Med. Biol. 510, 273-278 (2003).

11. S. J. Bailey et al., "Influence of repeated sprint training on pulmonary $\mathrm{O}_{2}$ uptake and muscle deoxygenation kinetics in humans," J. Appl. Physiol. 106(6), 1875-1887 (2009).

12. T. Hamaoka et al., "Near-infrared spectroscopy/imaging for monitoring muscle oxygenation and oxidative metabolism in healthy and diseased humans," J. Biomed. Opt. 12(6), 062105 (2007).

13. D. R. Lynch et al., "Near infrared muscle spectroscopy in patients with Friedreich's ataxia," Muscle Nerve 25(5), 664-673 (2002).

14. T. Okamoto et al., "Evaluation of oxygen uptake kinetics and oxygen kinetics of peripheral skeletal muscle during recovery from exercise in patients with chronic obstructive pulmonary disease," Clin. Physiol. Funct. Imaging 23(5), 257-262 (2003).

15. R. Belardinelli, "Muscle oxygenation kinetics measured by near-infrared spectroscopy during recovery from exercise in chronic heart failure," G. Ital. Cardiol. 28(8), 866-872 (1998).

16. M. Ferrari, M. Muthalib, and V. Quaresima, "The use of near-infrared spectroscopy in understanding skeletal muscle physiology: recent developments," Philos. Trans. R. Soc. London Ser. A 369(1955), 4577-4590 (2011). 
17. M. Wolf, M. Ferrari, and V. Quaresima, "Progress of near-infrared spectroscopy and topography for brain and muscle clinical applications," J. Biomed. Opt. 12(6), 062104 (2007).

18. B. Chance et al., "Optical investigations of physiology: a study of intrinsic and extrinsic biomedical contrast," Philos. Trans. R. Soc. London B 352(1354), 707-716 (1997).

19. Q. Zhang et al., "Study of near infrared technology for intracranial hematoma detection," J. Biomed. Opt. 5(2), 206-213 (2000).

20. J. Leon-Carrion et al., "The infrascanner, a handheld device for screening in situ for the presence of brain haematomas," Brain Inj. 24(10), 1193-1201 (2010).

21. T. Muehlemann, D. Haensse, and M. Wolf, "Wireless miniaturized invivo near infrared imaging," Opt. Express 16(14), 10323-10330 (2008).

22. B. Jones, C. M. Hesford, and C. E. Cooper, "The use of portable NIRS to measure muscle oxygenation and haemodynamics during a repeated sprint running test," Adv. Exp. Med. Biol. 789, 185-191 (2013).

23. Q. Zhang, X. Yan, and G. E. Strangman, "Development of motion resistant instrumentation for ambulatory near-infrared spectroscopy," J. Biomed. Opt. 16(8), 087008 (2011).

24. H. Atsumori et al., "Noninvasive imaging of prefrontal activation during attention-demanding tasks performed while walking using a wearable optical topography system," J. Biomed. Opt. 15(4), 046002 (2010).

25. H. Atsumori et al., "Development of wearable optical topography system for mapping the prefrontal cortex activation," Rev. Sci. Instrum. 80(4), 043704 (2009).

26. T. Hamaoka et al., "The use of muscle near-infrared spectroscopy in sport, health and medical sciences: recent developments," Philos. Trans. R. Soc. London Ser. A 369(1955), 4591-4604 (2011).

27. S. Koga et al., "Validation of a high-power, time-resolved, near-infrared spectroscopy system for measurement of superficial and deep muscle deoxygenation during exercise," J. Appl. Physiol. 118(11), 1435-1442 (2015).

28. V. Quaresima et al., "Nonuniform quadriceps $\mathrm{O}_{2}$ consumption revealed by near infrared multipoint measurements," Biochem. Biophys. Res. Commun. 285(4), 1034-1039 (2001).

29. H. Miura et al., "Relationship between muscle architectural features and oxygenation status determined by near infrared device," Eur. J. Appl. Physiol. 91(2-3), 273-278 (2004).

30. K. K. McCully, "The influence of passive stretch on muscle oxygen saturation," Adv. Exp. Med. Biol. 662, 317-322 (2010).

31. U. Wolf et al., "Regional differences of hemodynamics and oxygenation in the human calf muscle detected with near-infrared spectrophotometry," J. Vasc. Interventional Radiol. 18(9), 1094-1101 (2007).

32. J. P. Neary, "Application of near infrared spectroscopy to exercise sports science," Can. J. Appl. Physiol. 29(4), 488-503 (2004).

33. M. A. Khalil et al., "Detection of peripheral arterial disease within the foot using vascular optical tomographic imaging: a clinical pilot study," Eur. J. Vasc. Endovascular Surg. 49(1), 83-89 (2015).

34. A. LeBlanc et al., "Muscle volume, MRI relaxation times (T2), and body composition after spaceflight," J. Appl. Physiol. 89(6), 2158-2164 (2000).

35. J. J. Widrick et al., "Functional properties of slow and fast gastrocnemius muscle fibers after a 17-day spaceflight," J. Appl. Physiol. 90(6), 2203-2211 (2001).

36. K. M. Baldwin, "Effect of spaceflight on the functional, biochemical, and metabolic properties of skeletal muscle," Med. Sci. Sports Exercise 28(8), 983-987 (1996).

37. S. M. Smith et al., "Benefits for bone from resistance exercise and nutrition in long-duration spaceflight: evidence from biochemistry and densitometry," J. Bone Miner. Res. 27(9), 1896-1906 (2012).

38. F. J. DiMenna, S. J. Bailey, and A. M. Jones, "Influence of body position on muscle deoxy[Hb+Mb] during ramp cycle exercise," Respir. Physiol. Neurobiol. 173(2), 138-145 (2010).

39. B. Grassi et al., "Muscle oxygenation and pulmonary gas exchange kinetics during cycling exercise on-transitions in humans," J. Appl. Physiol. 95(1), 149-158 (2003).

40. S. Nioka et al., "Muscle deoxygenation in aerobic and anaerobic exercise," Adv. Exp. Med. Biol. 454, 63-70 (1998).

41. Q. Zhang, E. N. Brown, and G. E. Strangman, "Adaptive filtering for global interference cancellation and real-time recovery of evoked brain activity: a Monte Carlo simulation study," J. Biomed. Opt. 12(4), 044014 (2007).
42. Q. Zhang, E. N. Brown, and G. E. Strangman, "Adaptive filtering to reduce global interference in evoked brain activity detection: a human subject case study," J. Biomed. Opt. 12(6), 064009 (2007).

43. G. E. Strangman, Q. Zhang, and Z. Li, "Scalp and skull influence on near infrared photon propagation in the Colin27 brain template," NeuroImage 85(1), 136-149 (2014).

44. G. E. Strangman, Z. Li, and Q. Zhang, "Depth sensitivity and sourcedetector separations for near infrared spectroscopy based on the Colin27 brain template," PLoS One 8(8), e66319 (2013).

45. T. Funane et al., "Concurrent fNIRS-fMRI measurement to validate a method for separating deep and shallow fNIRS signals by using multidistance optodes," Neurophotonics 2(1), 015003 (2015).

46. M. Kiguchi and T. Funane, "Algorithm for removing scalp signals from functional near-infrared spectroscopy signals in real time using multidistance optodes," J. Biomed. Opt. 19(11), 110505 (2014).

47. I. Mizeva, P. Frick, and S. Podtaev, "Skin blood flow and temperature oscillations during cold pressor test," in 37th Annual Int. Conf. of the IEEE Engineering in Medicine and Biology Society (EMBC '16), pp. 7382-7385 (2015).

48. J. S. Petrofsky, "Resting blood flow in the skin: does it exist, and what is the influence of temperature, aging, and diabetes?" J. Diabetes Sci. Technol. 6(3), 674-685 (2012).

49. B. Page, 12-Lead ECG Placement for Acute and Critical Care Providers, Prentice Hall, Upper Saddle River, New Jersey (2006).

50. J. Maltez et al., "Time course and variability of power in different frequency bands of EEG during resting conditions," Neurophysiol. Clin. 34(5), 195-202 (2004).

51. M. Cope et al., "Methods of quantitating cerebral near infrared spectroscopy data," in Advances in Experimental Medicine and Biology, Vol. 222, pp. 183-189 (1988).

52. Y. Xu et al., "Image quality improvement via spatial deconvolution in optical tomography: time-series imaging," J. Biomed. Opt. 10(5), 051701 (2005).

53. C. H. Schmitz et al., "Instrumentation for fast functional optical tomography," Rev. Sci. Instrum. 73(2), 429-439 (2002).

54. Q. Zhang et al., "Experimental comparison of using continuous-wave and frequency-domain diffuse optical imaging systems to detect heterogeneities," Proc. SPIE 4250, 219 (2001).

55. Q. Zhang et al., "Coregistered tomographic x-ray and optical breast imaging: initial results," J. Biomed. Opt. 10(2), 024033 (2005).

56. D. A. Boas, "Photon migration imaging toolbox," 2004, http://www. nmr.mgh.harvard.edu/PMI/resources/tmcimg/index.htm (21 July 2016).

57. F. Menotti et al., "The role of the prefrontal cortex in the development of muscle fatigue in Charcot-Marie-Tooth 1A patients," Neuromuscular Disord. 24(6), 516-523 (2014).

58. S. Racinais et al., "Muscle deoxygenation and neural drive to the muscle during repeated sprint cycling," Med. Sci. Sports Exercise 39(2), 268-274 (2007).

59. H. L. Graber et al., "Spatiotemporal imaging of vascular reactivity," Proc. SPIE 3978, 364 (2000).

60. G. Strangman et al., "A quantitative comparison of simultaneous BOLD fMRI and NIRS recordings during functional brain activation," Neurolmage 17(2), 719-731 (2002).

61. R. Saager and A. Berger, "Measurement of layer-like hemodynamic trends in scalp and cortex: implications for physiological baseline suppression in functional near-infrared spectroscopy," J. Biomed. Opt. 13(3), 034017 (2008).

62. Q. Zhang, G. E. Strangman, and G. Ganis, "Adaptive filtering to reduce global interference in non-invasive NIRS measures of brain activation: how well and when does it work?" Neurolmage 45(3), 788-794 (2009).

63. R. B. Saager and A. J. Berger, "Direct characterization and removal of interfering absorption trends in two-layer turbid media," J. Opt. Soc. Am. A 22(9), 1874-1882 (2005).

64. G. Gratton and P. M. Corballis, "Removing the heart from the brain: compensation for the pulse artifact in the photon migration signal," Psychophysiology 32(3), 292-299 (1995).

65. E. M. Hillman et al., "Time resolved optical tomography of the human forearm," Phys. Med. Biol. 46(4), 1117-1130 (2001).

66. F. Felici et al., "Biceps brachii myoelectric and oxygenation changes during static and sinusoidal isometric exercises," J. Electromyogr. Kinesiol. 19(2), e1-e11 (2009). 
67. M. Muthalib et al., "Reliability of near-infrared spectroscopy for measuring biceps brachii oxygenation during sustained and repeated isometric contractions," J. Biomed. Opt. 15(1), 017008 (2010).

68. V. Quaresima et al., "Spatial distribution of vastus lateralis blood flow and oxyhemoglobin saturation measured at the end of isometric quadriceps contraction by multichannel near-infrared spectroscopy," J. Biomed. Opt. 9(2), 413-420 (2004).

69. E. Ohmae et al., "Sensitivity correction for the influence of the fat layer on muscle oxygenation and estimation of fat thickness by time-resolved spectroscopy," J. Biomed. Opt. 19(6), 067005 (2014).

70. E. Gerz et al., "Tissue oxygenation during exercise measured with NIRS: reproducibility and influence of wavelengths," Adv. Exp. Med. Biol. 789, 171-177 (2013).

71. K. K. McCully, C. Halber, and J. D. Posner, "Exercise-induced changes in oxygen saturation in the calf muscles of elderly subjects with peripheral vascular disease," J. Gerontol. 49(3), B128-B134 (1994).

72. J. R. Wilson et al., "Noninvasive detection of skeletal muscle underperfusion with near-infrared spectroscopy in patients with heart failure," Circulation 80(6), 1668-1674 (1989).

73. G. Giannotti et al., "Utility of near-infrared spectroscopy in the diagnosis of lower extremity compartment syndrome," J. Trauma 48(3), 396-399 (2000).

74. M. Scheuermann-Freestone et al., "Abnormal cardiac and skeletal muscle energy metabolism in patients with type 2 diabetes," Circulation 107(24), 3040-3046 (2003).

75. M. J. Christie, "Electrodermal activity and the stress response. A review," Acta Med. Pol. 14(4), 343-355 (1973).

76. N. Virag et al., "Prediction of vasovagal syncope from heart rate and blood pressure trend and variability: experience in 1,155 patients," Heart Rhythm 4(11), 1375-1382 (2007).

77. G. Themelis et al., "Near-infrared spectroscopy measurement of the pulsatile component of cerebral blood flow and volume from arterial oscillations," J. Biomed. Opt. 12(1), 014033 (2007).

78. B. P. Wagner and J. Pfenninger, "Dynamic cerebral autoregulatory response to blood pressure rise measured by near-infrared spectroscopy and intracranial pressure," Crit. Care Med. 30(9), 2014-2021 (2002).

79. R. Takizawa et al., "Anxiety and performance: the disparate roles of prefrontal subregions under maintained psychological stress," Cereb. Cortex 24(7), 1858-1866 (2014).

80. C. Bogler et al., "Decoding vigilance with NIRS," PLoS One 9(7), e101729 (2014).

81. D. J. Davies et al., "Near-infrared spectroscopy in the monitoring of adult traumatic brain injury: a review," J. Neurotrauma 32(13), 933-941 (2015).
82. P. A. Gould et al., "Investigating syncope: a review," Curr. Opin. Cardiol. 21(1), 34-41 (2006).

83. J. J. Engel et al., Epilepsy: A Comprehensive Textbook, LippincottRaven, Philadelphia, Pennsylvania (2007).

84. R. T. Higashida et al., "Trial design and reporting standards for intraarterial cerebral thrombolysis for acute ischemic stroke," Stroke 34(8), e109-e137 (2003).

85. C. O. Olopade et al., "Noninvasive determination of brain tissue oxygenation during sleep in obstructive sleep apnea: a near-infrared spectroscopic approach," Sleep 30(12), 1747-1755 (2007).

Gang $\mathrm{Hu}$ is a biomedical engineer and instructor at Harvard Medical School and Massachusetts General Hospital. He has 10 years of experience developing innovative devices for biomedical applications. In the past two years, he focused on technology development for multimodal, wearable neuro-monitoring, and neuroimaging.

Quan Zhang is an instructor in psychology at Harvard Medical School and the director of the Biomedical Engineering Lab at the Neural Systems Group, Massachusetts General Hospital. He has 20 years of experience developing innovative devices for biomedical applications. His recent research includes wearable neuromonitoring and neuroimaging, and their applications in various clinical and space applications.

Vladimir Ivkovic is a postdoc research fellow at Massachusetts General Hospital and Harvard Medical School. He is a cognitive neuroscientist with more than ten years of research experience on neuromonitoring and sensory augmentation in elite (astronaut) and clinical (Parkinson's disease) populations. He currently focuses on the applications of long-term wearable neuromonitoring and neuroimaging for clinical and extreme environments. He has been a Co-I on multiple research grants, including NASA and CNES parabolic flight studies.

Gary E. Strangman is an associate professor in the Department of Psychiatry at Harvard Medical School, director of the Neural Systems Group at Massachusetts General Hospital, and team leader of the National Space Biomedical Research Institute's Smart Medical Systems and Technology team. His research focuses on translational neuroscience, developing novel neuroimaging technologies for use in research, clinical and operational environments. 